\title{
Article \\ A Flexible and Stretchable Self-Powered Nanogenerator in Basketball Passing Technology Monitoring
}

\author{
Changjun Jia ${ }^{1}\left(\mathbb{D}\right.$, Yongsheng Zhu ${ }^{1}$, Fengxin Sun ${ }^{1}$, Tianming Zhao ${ }^{2} \oplus$, Rongda Xing ${ }^{3}$, Yupeng Mao ${ }^{1, *(1)}$ \\ and Chongle Zhao ${ }^{1, *(D)}$ \\ 1 Physical Education Department, Northeastern University, Shenyang 110819, China; \\ 2071367@stu.neu.edu.cn (C.J.); 2001276@stu.neu.edu.cn (Y.Z.); 2171435@stu.neu.edu.cn (F.S.) \\ 2 College of Sciences, Northeastern University, Shenyang 110819, China; zhaotm@stumail.neu.edu.cn \\ 3 College of Information Science and Engineering, Northeastern University, Shenyang 110819, China; \\ 20194324@stu.neu.edu.cn \\ * Correspondence: maoyupeng@pe.neu.edu.cn (Y.M.); zhaochongle@pe.neu.edu.cn (C.Z.)
}

Citation: Jia, C.; Zhu, Y.; Sun, F.; Zhao, T.; Xing, R.; Mao, Y.; Zhao, C. A Flexible and Stretchable Self-Powered Nanogenerator in Basketball Passing Technology Monitoring. Electronics 2021, 10, 2584. https://doi.org/ 10.3390 /electronics10212584

Academic Editor: Massood Tabib Azar

Received: 19 September 2021

Accepted: 19 October 2021

Published: 22 October 2021

Publisher's Note: MDPI stays neutral with regard to jurisdictional claims in published maps and institutional affiliations.

Copyright: (c) 2021 by the authors Licensee MDPI, Basel, Switzerland. This article is an open access article distributed under the terms and conditions of the Creative Commons Attribution (CC BY) license (https:// creativecommons.org/licenses/by/ $4.0 /)$.

\begin{abstract}
The rapid development of the fifth generation technology poses more challenges in the human motion inspection field. In this study, a nanogenerator, made by PVDF, ionic hydrogel, and PDMS, is used. Furthermore, a transparent, stretchable, and biocompatible PENG (TSB-PENG) is presented, which can be used as a self-powered sensor attached to the athlete's joints, which helps to monitor the training and improve the subject's performance. This device shows the ability to maintain a relatively stable output, under various external environments (e.g., inorganic salt, organic matter and temperature). Additionally, TSB-PENG can supply power to small-scale electronic equipment, such as Bluetooth transmitting motion data in real time. This study can provide a new approach to designing lossless, real-time, portable, and durable self-powered sensors in the sports motoring field.
\end{abstract}

Keywords: flexible; self-powered; sport monitoring; basketball pass

\section{Introduction}

The world is entering the era of Internet of Things (IoT) at an accelerated speed, based on the rapid development of the fifth generation technology [1-4]. Wearable electronic equipment and sensors are increasingly developing over the past few years and have shown good application prospects in many fields [5-8]. In the sports field, the recording and monitoring of human motion training data bear great importance to the promotion of the sports development. At the same time, they are important research objects, in the field of sports training [9-13]. According to known research achievements, wearable electronic equipment has already been applied to the theory and practice of sports training, such as basketball, athletics and swimming [14-20]. The monitoring of relevant data of athletes, such as physiological, biochemical, and anatomical data, has been implemented, in order to improve the athletic performance. Specifically, in basketball, the winning factors are mainly the transformation of attack and defense [21,22]. It is manifested as the center player's pivot teamwork, perfect passing, and dribbling skills, as well as the cooperation level of the whole team, where the center player's passing method is particularly important to the game [23-25]. Consequently, the monitoring for the center player's passing method is an urgent problem requiring solution. In some existing studies, audio/video and virtual reality equipment are used to monitor the training process of the center player [26-29]. However, these equipment pieces are usually large in size and not adjustable, while the maintenance costs are high, operation is complex, and external power supply is required, posing potential dangers. In the field of wearable electronic products development and application research, the rechargeable lithium battery use is always a problem [30,31], which the development of a biocompatible, comfortable and stable sensor, for real-time human physiological monitoring, is called to solve. 
At present, piezoelectric materials are widely used in the sensor hardware and energy collection field [32-35]. The traditional sensors, based on the piezoelectric effect, such as piezoelectric ceramics, lead zirconate titanate, and niobate lead magnesium, have not been widely used as wearable human motion monitoring sensors, due to brittleness, deleteriousness, complex manufacturing, and tendency to corrosion [36-40]. This kind of sensors should have mechanical flexibility and transparency, so as to adapt to the strain of the human skin and joints and easily observe the state of human joints in motion. Moreover, the biocompatibility of PENG sensors, in direct contact with human body, should be an indicator to be considered. It must be nontoxic and does not cause the body's immune response. In addition, considering that, during sports training, the rise of the body temperature generates a lot of sweat, consisting of different concentrations of inorganic salts and urea, as well as other organic matters, which may lead to electronic equipment circuit corrosion, damaging the PENG [41-43]. Hence, the PENG used for human motion monitoring should have soft, transparent, biocompatible and durable features.

In this study, we present a piezoelectric nanogenerator constituted by PVDF, ionic hydrogel and PDMS. Different from the previously reported PENG using metal as electrodes, this kind of soft and transparent PENG (TSB-PENG) uses lithium chloride polyacrylamide hydrogel as electrodes. This piezoelectric nanogenerator, based on PAAM-LiCl, has the advantages of high transparency, softness, non-invasiveness, good biocompatibility, anticorrosion, and simple fabrication. The PENG attached on human skin surface will not affect the comfort and can be stretched according to the changes of body joint angles. Moreover, it can resist more severe environment erosion and, thus, monitor the sports training under more secure and portable conditions. This transparent, soft, and biocompatible PENG can provide a novelty to the sports training monitoring.

\section{Experimental Section}

\subsection{Materials}

Poly (vinylidene fluoride) (PVDF) powder was bought from Qinshang plastic co., ltd (Suzhou, Jiangsu, China). N, N-Dimethylformamide (DMF); deionized water; acrylamide (AM); lithium chloride ( $\mathrm{LiCl}) ; \mathrm{N}, \mathrm{N}^{\prime}$-methylene diacrylamide (MBA); ammonium persulphate (APS); and $\mathrm{N}, \mathrm{N}, \mathrm{N}^{\prime}, \mathrm{N}^{\prime}$-Tetramethylethylenediamine (TMLD) are bought from Jintong letai chemical industry products Co., Ltd. (Beijing, China). DOW CORNING 3140 RTV and Svlgard 184 were bought from Xinheng trading Co., Ltd. (Tianjin, China). All purified materials were used directly and were not purified.

\subsection{Manufacture of Piezoelectric Nanogenerator}

The manufacture of PVDF piezoelectric film is as follows. Firstly, 15\% and 85\% PVDF powder and DMF solution were stirred in a $60{ }^{\circ} \mathrm{C}$ water bath for $2 \mathrm{~h}$. Semi-transparent mixture solution was set in a vacuum drying oven for $30 \mathrm{~min}$ to remove bubbles. Secondly, the mixture solution was dropped on the bottom silicon rotating surface of the spin coater which spun in $400 \mathrm{r} / \mathrm{min}$ for $20 \mathrm{~s}$. Then, PVDF/MBA mixture was dried at $80{ }^{\circ} \mathrm{C}$ for $15 \mathrm{~min}$. After the second step was repeated three times, it was dried at $120{ }^{\circ} \mathrm{C}$ for $12 \mathrm{~h}$, and the multilayer film was prepared. Finally, films were dealt with polarization which was at $90{ }^{\circ} \mathrm{C}$ and $20 \mathrm{kV} / \mathrm{mm}$ oil bath to gain PVDF piezoelectric films.

Synthesis of lithium chloride polyacrylamide hydrogel is as follows. AM was used as a monomer, MBA as a crosslinking agent, APS as an initiator, and TMEDA as a catalyst, and, finally, hydrogel obtained by AM polymerization. The specific steps were as follows. Firstly, acrylamide powder and lithium chloride particles were dissolved in $50 \mathrm{~mL}$ of water at a speed of $600 \mathrm{rpm}$. The concentrations of PAAM and lithium chloride were $3 \mathrm{~mol} / \mathrm{L}$ and $5 \mathrm{~mol} / \mathrm{L}$, respectively. MBA and APS were added to the solution, and the weights were 0.02 and $0.03 \mathrm{~mol} \%$, respectively. The particles were stirred until they were completely dissolved, and then rested for $10 \mathrm{~min}$ to obtain the pre solution. Secondly, a few drops of TMEDA $(0.0025 \mathrm{wt} \%)$ were dropped in pre-solution and stirred to accelerate 
commissure for the hydrogel. Then, the mixture solution was dropped in culture plate to gain PAAM-LiCl hydrogel.

The manufacturing method of transparent and soft Peng is as follows. Firstly, the edges of PVDF film were treated with Dow Corning 3140 RTV to prevent short circuit, and stood for $30 \mathrm{~min}$. Secondly, we put the mixture of Sylgard 184 and curing agent (the weight ratio of 20:1) on the groove model (produced by $3 \mathrm{~d}$ printing). Then, we cured in the drying oven at $80^{\circ} \mathrm{C}$ for $20 \mathrm{~min}$. Thirdly, according to the actual needs, we cut hydrogel with the appropriate size. Finally, double pieces of the hydrogel were put on both sides of the PVDF film to prevent short-circuit. Then, the polyvinylidene fluoride film was packaged by PDMS and adhered to by Dow Corning 3140 RTV.

\subsection{Characterization and Measurement}

The transparent and soft PENG was fixed on the stepping motor to simulate joint movement. The different amplitudes and frequencies were used to test PENG characteristics. Piezoelectric signals were generated by sensors and collected by oscilloscopes (sto 1102 c, Shenzhen, China). The morphology and structure of the sensor were carried out by an optical microscope (Sunshine Instrument Co., Ltd., SDPTOP-CX 40 m, Ningbo, China).

\section{Results}

As shown in Figure 1a, PENG can be applied to the training of basketball athlete. Based on the piezoelectric effect, the piezoelectric signal of TSB-PENG is a sensing signal. It is small and transparent and can be attached to the joint surface of the athlete for collecting and analyzing sport information. The body motion is the kind of three-dimensional movement, and every joint motion is along or parallel to the sagittal plane, anterior plane and parallel plane. At the same time, the completion of human action is a complex joint motion, such as spinning forward and backward, twisting, and pulling. Therefore, the soft and stretchable sensor is more suitable for monitoring human motion. Figure $1 \mathrm{~b}-\mathrm{d}$ show that TSB-PENG can work at states of bend, twist, and stretch, and can monitor the joint motion with comfort. The piezoelectric signal of the above three states can be learned in Movie S1. Figure 1e shows the transmittance of PVDF film, PAAM-LiCl hydrogel, PDMS, and TSB-PENG. The average light transmittances of the PDMS, PVDF, and PAAM-LiCl hydrogels were above $86 \%$. The average light transmittances of the PDMS, PAAM-LiCl hydrogel, and PVDF film were $94.32,92.36$, and $86.96 \%$. Figure $1 \mathrm{f}-\mathrm{h}$ show the optical microscope images of the PVDF film, PAAM-LiCl hydrogel, and PDMS under 258 times. The conductivity of PAAM- $\mathrm{LiCl}$ hydrogel is $7.73 \mathrm{k} \Omega / \mathrm{cm}$. In addition, mechanical flexibility, extendibility, transparency, and durability are important to a sensor for monitoring body motion. Compared with metal electrodes, the advantages are shown in Supplementary Material Table S1. The manufacturing process of TSB-PENG is shown in Figure 1i. According to practical needs, we cut the suitably sized hydrogel. Dow Corning 3140 RTV is used to prevent the short-circuit for PVDF film (stage I). As the electrodes, double PAAM-LiCl hydrogels were covered on both sides of the PVDF film (stage II). Thereafter, PDMS layers were used as protective layers (stage III). Then, 3140 glue was used to seal. Finally, it was placed at room temperature for $24 \mathrm{~h}$, and then the final TSB-PENG was obtained. 


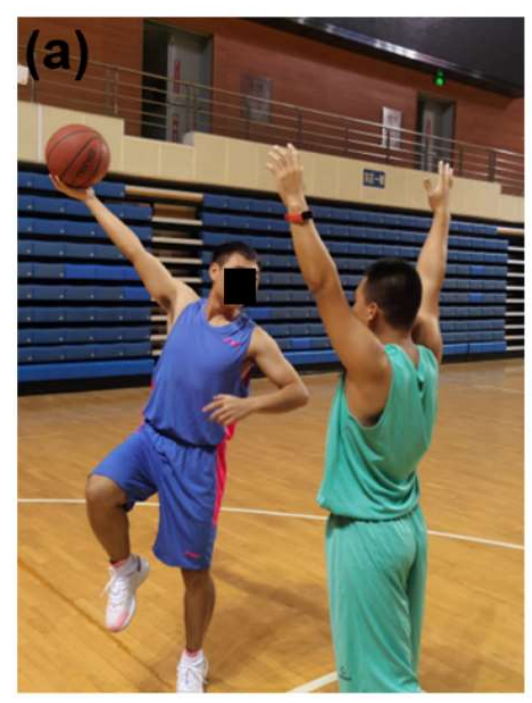

(b)

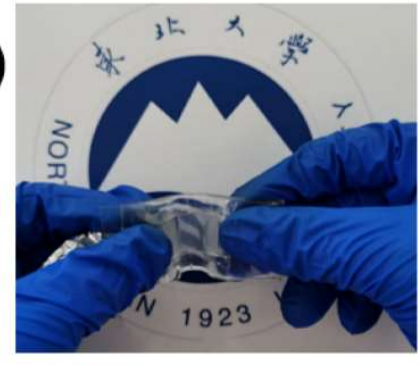

(d)
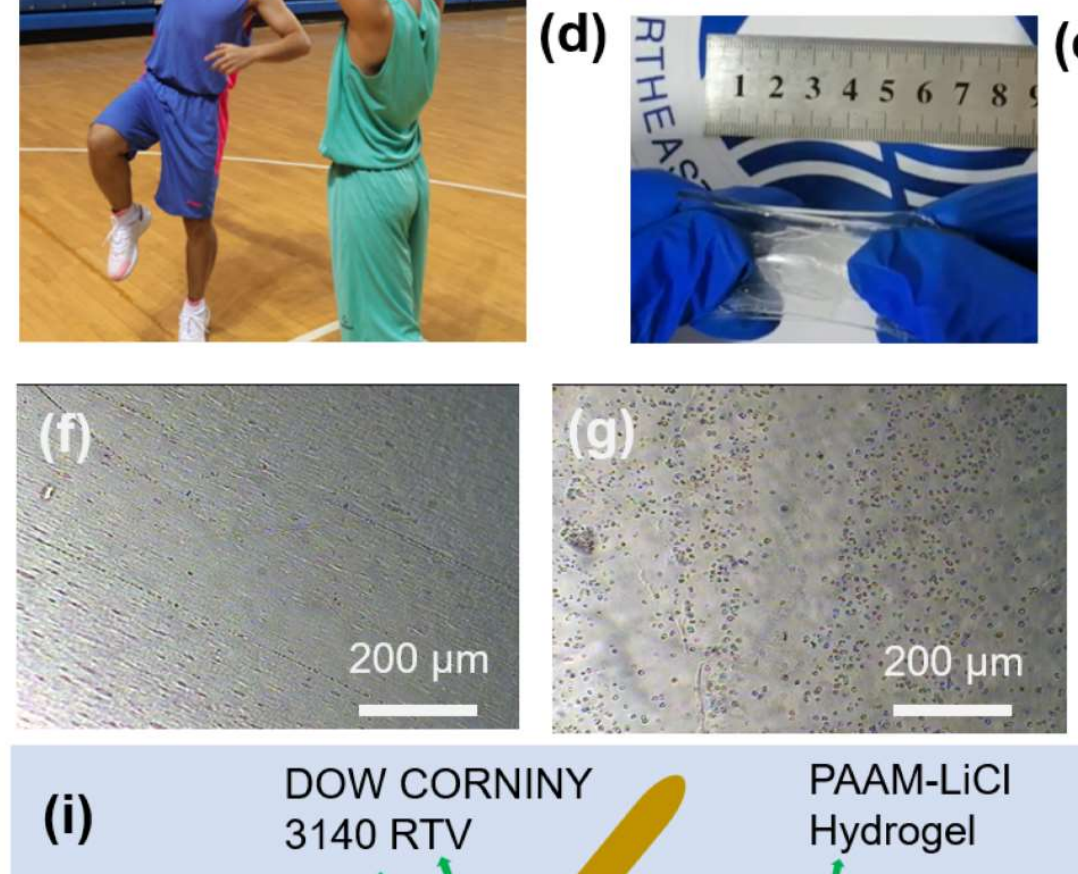

(i)

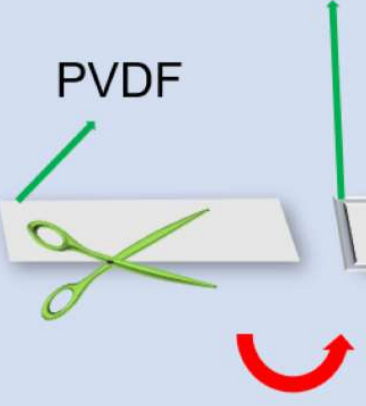

I
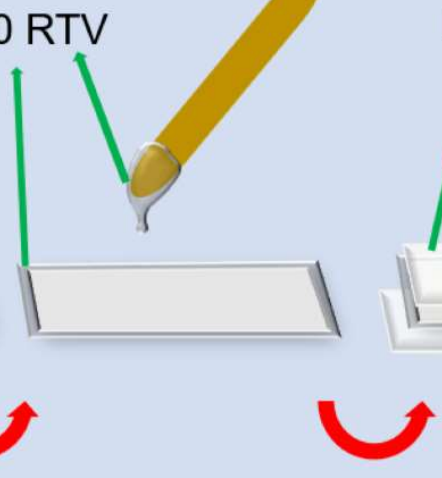

II

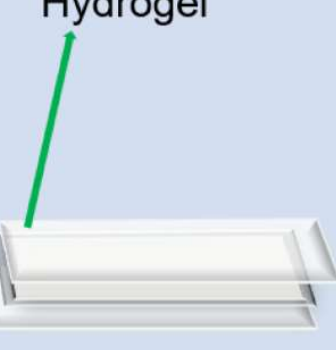

(c)

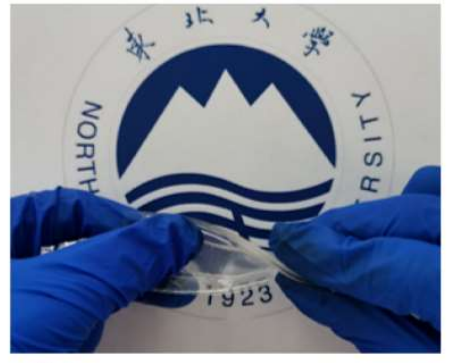

(e)
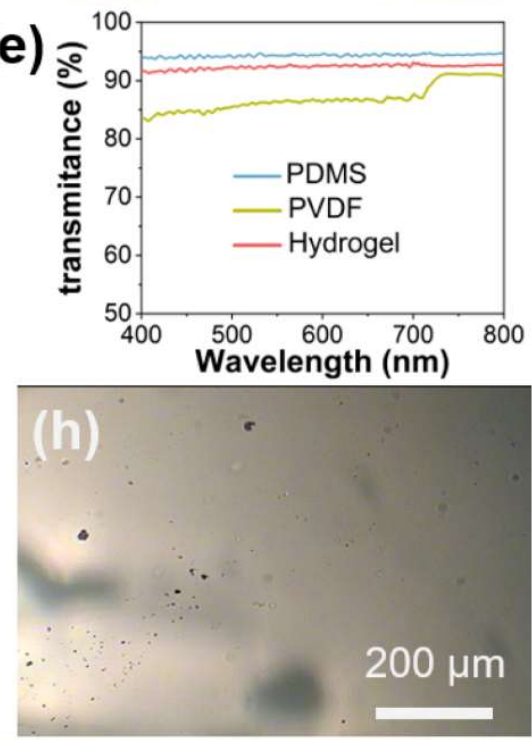

PAAM-LiCl
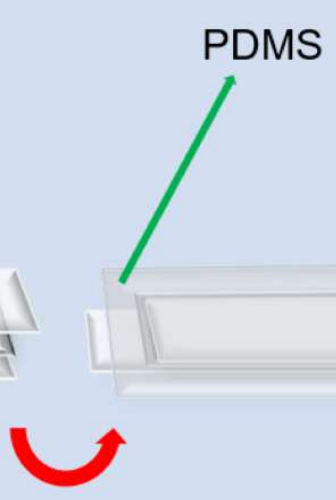

III

Figure 1. (a) The actual application scenarios of TSB-PENG. (b-d) The bend, twist and stretch state of TSB-PENG (e) The transmittance of PVDF film, PAAM-LiCl hydrogel, PDMS, and TSB-PENG. (f-h) The optical microscopic image of PVDF film, PAAM-LiCl hydrogel, and PDMS. (i) Schematic diagram of manufacturing process of TSB-PENG.

The working mechanism of TSB-PENG shows in Figure 2a. When the deformation has not occurred on PVDF, dipoles ordered arrangement in PVDF and bound a large amount of charge. When PVDF is deformed, dipoles are in disordered arrangement, the electric charge is released, and the voltage/electric current is detected. When the external force disappears, dipoles return to the orderly arrangement, the charge is bound again, and the opposite voltages/currents are detected. In Figure 2b, we tested TSB-PENG properties. The TSB-PENG, with a size of $5 \mathrm{~cm} \times 2 \mathrm{~cm}$, was fixed on the stepper motor to imitate body joint motion. Figure $2 c$ shows the outputting piezoelectric of the TSB-PENG at the same frequency $(1 \mathrm{~Hz})$ and different bend angles. When the angle is $175,166,152$, and $138^{\circ}$, the outputting piezoelectric voltage is $0.54,0.87,1.54$, and $1.99 \mathrm{~V}$, respectively. Figure $2 \mathrm{~d}$ shows 
the outputting piezoelectric voltage of the TSB-PENG at $1 \mathrm{~Hz}$ frequency and different twist angles. When the angle is $20,35,50$, and $65^{\circ}$, the outputting piezoelectric voltage is $0.9,1.5$, 2.4 , and $4.1 \mathrm{~V}$, respectively. The relation of outputting piezoelectric voltage with frequency is shown in Figure 2e. When the bend angle is at the constant angle $\left(140^{\circ}\right)$, the frequency is $0.5,1,1.5$, and $2 \mathrm{~Hz}$, and the outputting piezoelectric voltage is $1.85,1.88,1.86$, and $1.92 \mathrm{~V}$, respectively. This shows that the outputting piezoelectric voltage is stable. Figure $2 \mathrm{f}$ shows the response of TSB-PENG is at different bend angles, twist angles, and frequencies. The response of TSB-PENG can be calculated from the following equation:

$$
R \%=\left|\frac{V_{0}-V_{i}}{V_{i}}\right| \times 100 \%
$$

where $V_{0}$ and $V_{i}$ are the output and piezoelectric voltages, respectively. When the TSBPENG is at different bend angles, the response of TSB-PENG is 0, 38.5, 65.1, and 73.1\%. When the TSB-PENG is at different twist angles, the response of TSB-PENG is 0, 15.3, 61.9, and $78.3 \%$, and when the frequency is $0.5,1,1.5$ and $2 \mathrm{~Hz}$, the response of TSB-PENG is $0,1.5,0.6$ and $3.9 \%$. It shows that TSB-PENG can monitor the tester's motion angle and frequency changes. These data can be used to analyze the state of basketball athletes to analyze their technical performance.

In order to prove that TSB-PENG can be applied to the body surface directly, the TSB-PENG is fixed on the body surface for $6 \mathrm{~h}$ and no allergic or rejection happens. Human sweat contains a large number of compounds, such as sodium chloride, potassium, and nitrogen metabolites (urea, ammonia, and uric acid) [20]. In this study, different concentrations of $\mathrm{NaCl}$ and urea are used to verify the impact on PENG (Figure $3 b, c)$. When the TSB-PENG is at $0.5,1,1.5$, and $2 \mathrm{~mol} / \mathrm{L}$ of $\mathrm{NaCl}$, the outputting piezoelectric voltage of TSB-PENG is $1.08,1.08,1.12$, and $1.23 \mathrm{~V}$, respectively. When the TSB-PENG is at $0.25,0.5$, 0.75 , and $1 \mathrm{~mol} / \mathrm{L}$ of urea, the outputting piezoelectric voltage of TSB-PENG is $1.48,1.52$, 1.45 , and $1.54 \mathrm{~V}$, respectively. The response of piezoelectric voltage in different $\mathrm{NaCl}$ and urea concentrations is stable. The stable response proves that the TSB-TENG is not affected by sweat. We test the outputting voltage of the sensor when it is at $28-40{ }^{\circ} \mathrm{C}$ (Figure $3 \mathrm{e}$ ). When the temperature is $28,30,32,34,36,38$, and $40^{\circ} \mathrm{C}$, the outputting piezoelectric voltage is $2.74,2.79,2.81,2.77,2.74,2.792$, and $2.78 \mathrm{~V}$, respectively. This shows that TSB-PENG has a good temperature adaptability. Figure $3 \mathrm{f}$ shows the durability property of TSB-PENG. The TSB-PENG was tested for $20 \mathrm{~min}$ and the piezoelectric voltage was stable. This shows that TSB-PENG can work for a long time. As shown in Supplementary Material Figure S1, the TSB-PENG output voltage is still stable at different angles and frequencies. This proves that TSB-PENG has perfect adaptability at different conditions.

Figure $4 \mathrm{a}-\mathrm{c}$ show that TSB-PENG, with a size of $5 \mathrm{~cm} \times 2 \mathrm{~cm}$, is fixed on the shoulder, elbow, and wrist of the athlete to monitor the different motion information. Figure $4 \mathrm{~d}$ shows the outputting piezoelectric voltage of TSB-PENG that is fixed on the shoulder. The shoulder joint is composed of the acromioclavicular joint, the sternoclavicular joint, the scapular chest walls joint, and the glen humeral joint. The shoulder joint is the most flexible in the human body. The motion of the shoulder joint is of bend and stretch. Therefore, the bend and stretch action signals are collected by us. Figure $4 \mathrm{e}, \mathrm{f}$ are the enlarged drawing of the bend and stretch motion of the shoulder. The motion of bend and stretch are the series of motions that are stretch recovery, bend recovery, and then stretch recovery. At the same time, the shape of piezoelectric voltage is as follows: down-up-down-up-down-back to the original position. This kind of motion is more complicated than extension-adduction movement. According to the comparison of the outputting piezoelectric voltage, the voltage of the shoulder is less than the voltage of the other joint. It is because the shoulder has a wide range of motion, many small joints make up the shoulder joint. When the shoulder moves, many small joints move together. The deformation that occurs on the TSB-PENG is small. Figure $4 \mathrm{~g}$ shows the outputting piezoelectric voltage of TSB-PENG is attached to the elbow. The elbow joint is a composite joint that is composed of the brachioulnar joint, the brachioradialis joint, and the proximal radioulnar joint. The brachialulnar joint 
is the main joint of the elbow joint, mainly performing bend and stretch motion. The proximal radioulnar joint is responsible for the rotation of the forearm. Figure $4 \mathrm{~h}$, i show the enlarged drawings of the elbow doing pronation and bend-stretch movements. In Figure $4 \mathrm{~h}$, it is obvious that the piezoelectric voltage goes up and down with pronation recovery motion. At the same time, the piezoelectric voltage fluctuates up and down with the bend and stretch action (Figure 4i). Because the action amplitude of bend and stretch is bigger than twist amplitude, the voltage of bend and stretch is bigger than the voltage of twist. Figure $4 \mathrm{j}$ shows that the outputting piezoelectric voltage of TSB-PENG is fixed on the wrist. The wrist joint consists of the radiocarpal joint (which makes the hand bend and stretch) and the interphalangeal joint (which makes the hand rotate back and forth). Figure $4 \mathrm{k}, 1$ show the enlarged drawings of the wrist doing protrusion and bend-stretch movements. These kinds of motion are similar to the elbow motion, therefore, the signal trend is similar also. The outputting piezoelectric voltage of the wrist is higher than that because wrist deformation is bigger. It proves that TSB-PENG can be used to monitor the information of body joint motion.

(a)

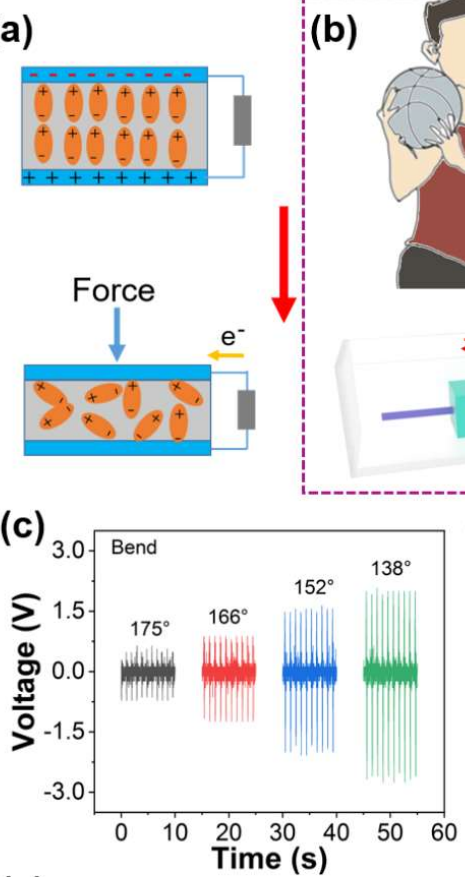

(e)

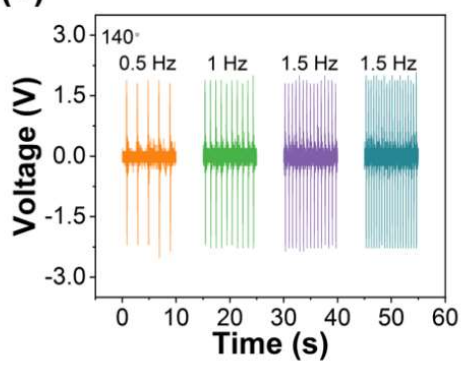

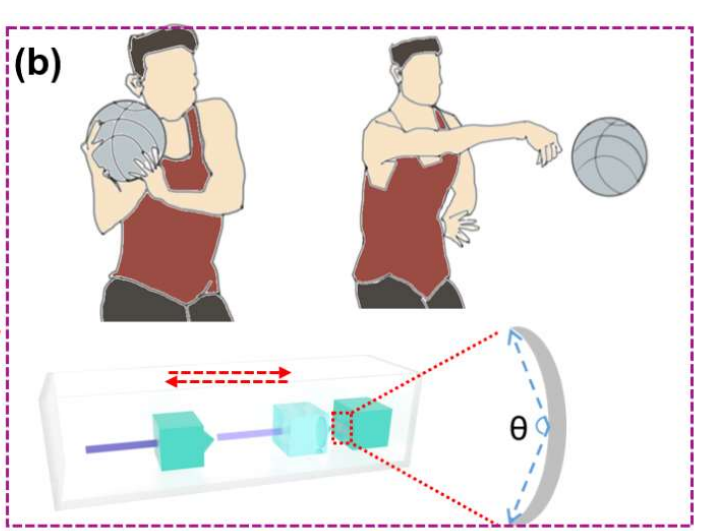

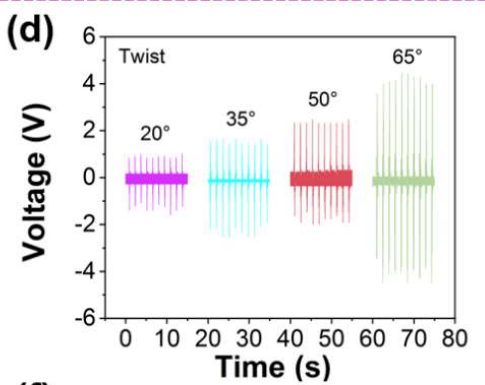

(f)

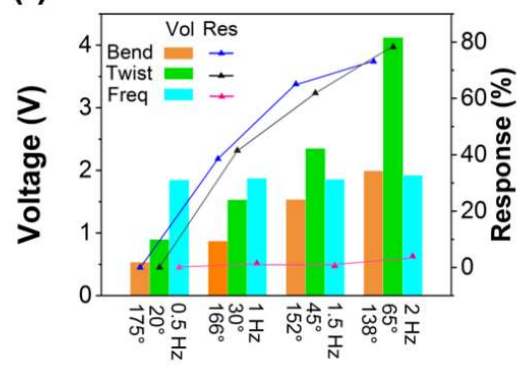

Figure 2. (a) Working mechanism of TSB-PENG; (b) Scene of TSB-PENG monitors the body joint motion system; (c) Outputting piezoelectric voltage of TSB-PENG at the same frequency and different bend angles; (d) Outputting piezoelectric voltage of TSB-PENG at the same frequency and different twist angles; (e) Outputting piezoelectric voltage of TSB-PENG at the same bend angle and different frequencies; (f) Response of output piezoelectric voltage of TSB-PENG at different bend angles, frequencies and twist angles. 
TSB-PENG can not only be used as a sensor to monitor athlete sport status, but it can also collect the mechanical energy of the human body. Figure 5a shows the equivalent circuit of the self-charge system and it can convert the mechanical energy of the human body into electric energy and store it in the capacitor. Figure 5b shows the charging voltage of different capacitor which is charged by TSB-PENG. When the bend angle is $150^{\circ}$ and the frequency is $3 \mathrm{~Hz}$, the TSB-PENG can charge $1,2.2$, and $3.3 \mu \mathrm{F}$ to $2.8,1.7$, and $0.3 \mathrm{~V}$. In Movie S2, TSB-PENG charges $4.7 \mu \mathrm{F}$ for $30 \mathrm{~s}$ and drives a portable calculator. Figure $5 c$,d show the TSB-PENG can drive Bluetooth. The transmitting terminal can launch the piezoelectric signal to receiving terminal. In the Movie S3, the number of flashing lights and flashing frequency is controlled by TSB-PENG. This wireless information transmission function provides broad application scenarios for TSB-PENG.
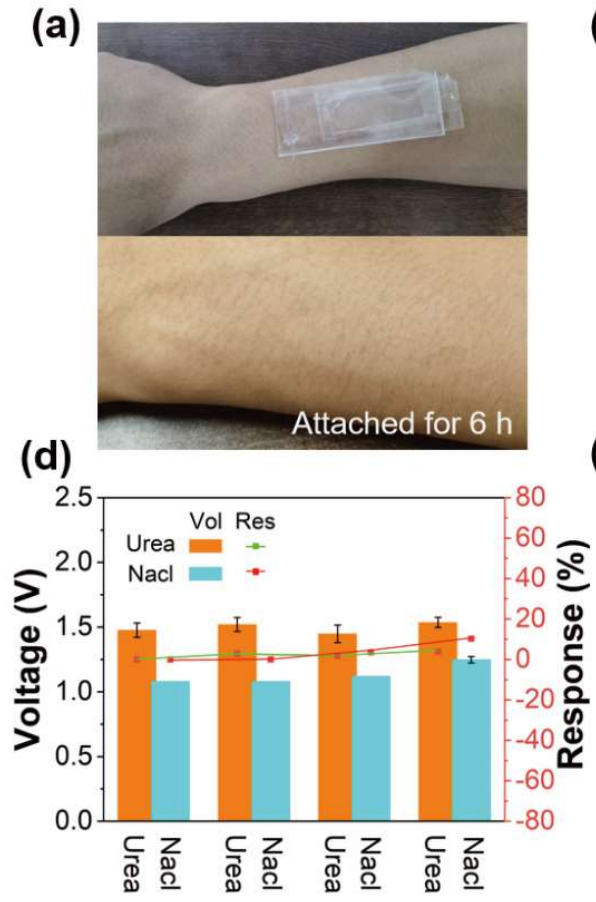

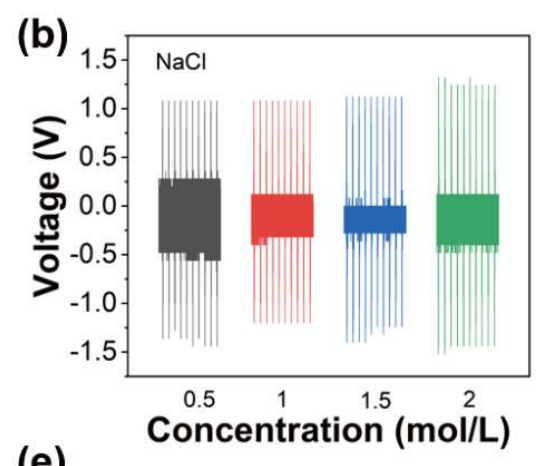

(e)

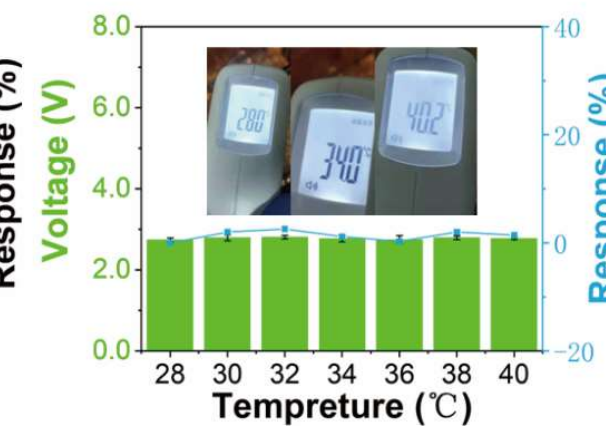

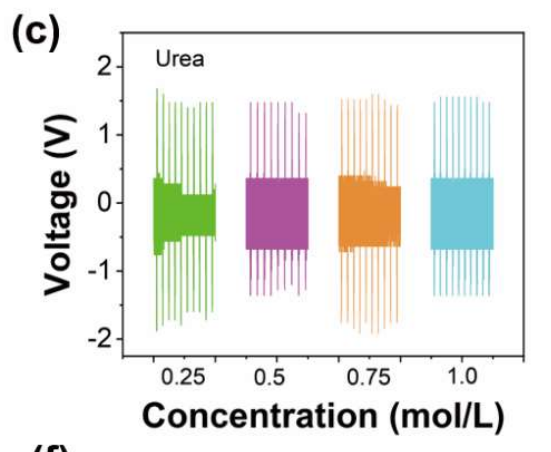

(f)

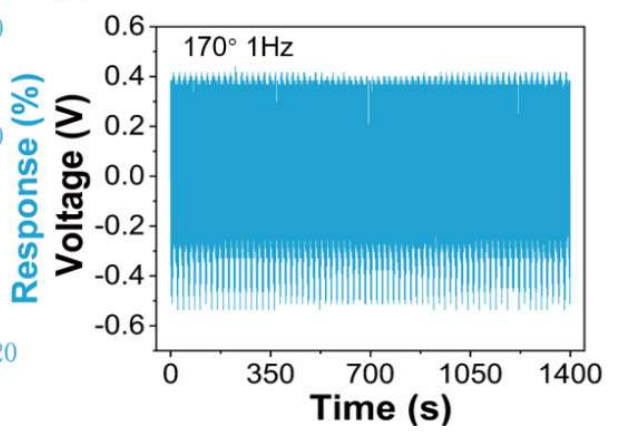

Figure 3. Physiological monitoring properties of TSB-PENG. (a) Image of TSB-PENG attaches to the arm for $6 \mathrm{~h}$. $(\mathbf{b}, \mathbf{c})$ Outputting piezoelectric voltage of TSB-PENG in different concentrations of $\mathrm{NaCl}$ and urea. (d) Response of outputting piezoelectric voltage of TSB-PENG is in different concentrations of $\mathrm{NaCl}$ and urea. (e) Outputting piezoelectric voltage of WSB-PENG is at different temperatures. (f) Durability property of TSB-PENG. 
(a)
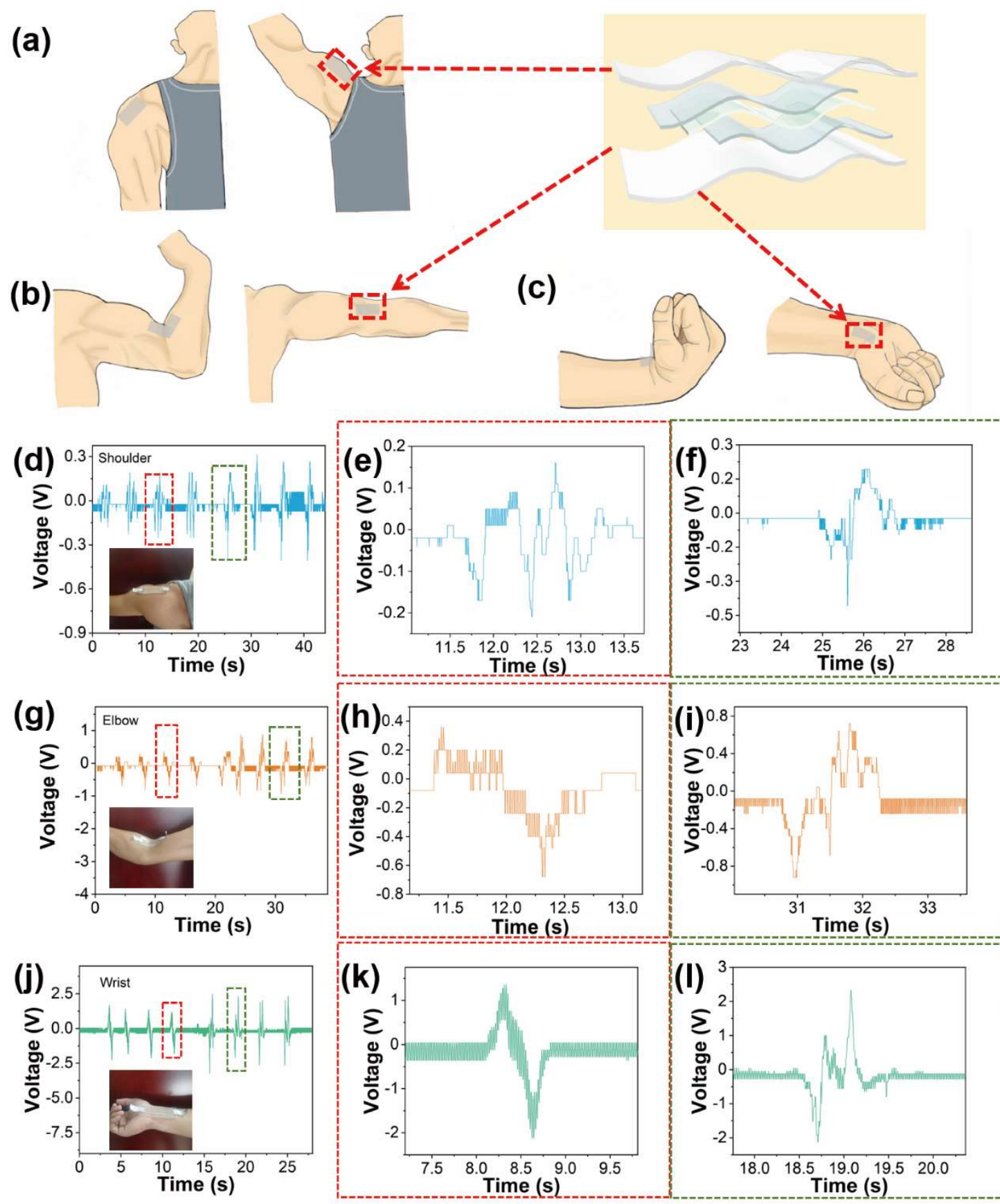

Figure 4. (a-c) Scenes of the working mechanism of TSB-PENG is fixed on the shoulder, elbow, and chest. (d) Outputting piezoelectric voltage of TSB-PENG on the shoulder that does the bend and stretch motion. (e,f) Enlarged drawings of bend and stretch motion of the shoulder. (g) Enlarged drawing of outputting piezoelectric voltage of TSB-PENG is fixed on the elbow which protrudes, bend and stretch. $(\mathbf{h}, \mathbf{i})$ Enlarged drawings of the elbow that does protrusion and bend-stretch movements. (j) Outputting piezoelectric voltage of TSB-PENG is fixed on the wrist that protrudes, bends, and stretches. $(\mathbf{k}, \mathbf{l})$ Enlarged drawings of the wrist in a protruding and bend stretch motion. 
(a)

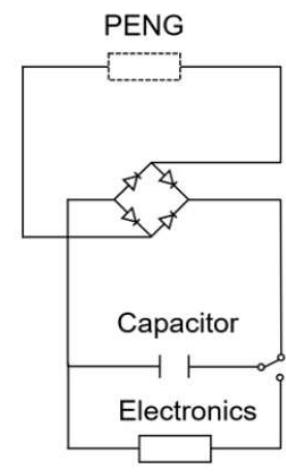

(b)

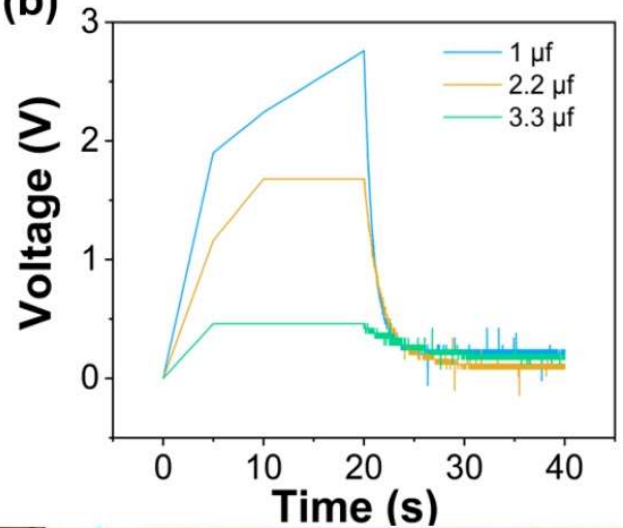

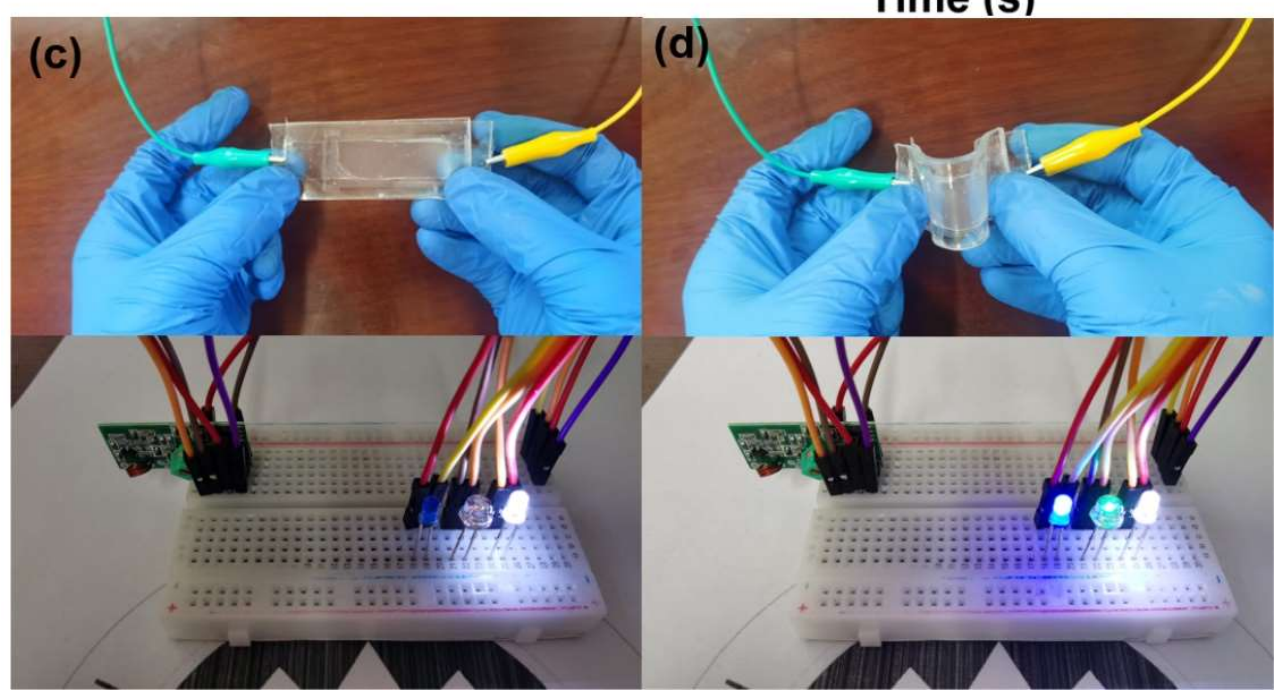

Figure 5. Feasibility of TSB-PENG as a power source. (a) Equivalent circuit of the self-charge system. (b) Charging voltage of different capacitor which is charged by TSB-PENG. (c,d) TSB-PENG drives Bluetooth device.

\section{Discussion}

Wearable electronic equipment was applied to sports training. It was found that traditional piezoelectric sensors cannot be used as wearable human motion monitoring sensors, owing to their brittleness, deleteriousness, complex manufacturing, and so on [36-40]. We made a transparent, stretchable, and biocompatible PENG, which was monitored in basketball passing technique. It can adapt to the environment of sport training and it can collect motion information of athletes. For example, when athletes do different motions, the peak of characteristic signals are different. According to the signal peak analysis, we can infer athletes' motions defect and correct them in time.

In the basketball game, the quality of passing is one of the decisive factors in the game. Accurate and reasonable passing can tear the opponent's defense and create the best shooting opportunities [44]. We analyze three kinds of passing techniques with examples, as shown in Figure 6. The outputting piezoelectric voltage of hook pass, shoulder pass, and chest pass are often used in the inner coping area, outer coping area, and middle coping area (The collection process is shown in Movies S4-S6). Figure 6a,e,i show the diagram of hook pass, shoulder pass, and chest pass, respectively. Figure $6 \mathrm{~b}-\mathrm{d}$ show the outputting piezoelectric voltages of the shoulder, elbow, and wrist in a hook pass motion which are $0.39,0.76$, and $2.77 \mathrm{~V}$, respectively. Figure $6 \mathrm{f}-\mathrm{h}$ show the outputting piezoelectric voltage of shoulder, elbow, and wrist in the shoulder pass motion which are $0.18,1.22$, and $3.564 \mathrm{~V}$, respectively. Figure $6 \mathrm{j}-1$ show the output piezoelectric voltages of the shoulder, elbow, and wrist in a chest pass motion, which are voltage is $0.83,1.61$, and $5.41 \mathrm{~V}$, respectively. The 
voltage of hook pass of the elbow and shoulder is smaller than the voltage of the other two passing of wrist. This is because the hook pass is the player who leads the ball over the head and buckle the wrist to pass the ball, the elbow participates less, and the voltage is lower. The shoulder pass motion requires the athlete to lead the ball over the shoulder, stretch the elbow, and buckle the wrist to pass the ball, whereby the shoulder participates less, and the voltage is lower. Compared with the outputting piezoelectric voltage of three passing types, the voltage of the hook pass is smaller than the other two. This is because the hook pass is usually used in internal coordination area and player passes the ball with the fast motion. The outputting piezoelectric voltage of the chest past in the central response area is higher than the shoulder. This is because the chest pass is fast and accurate in the central response area and the player needs to pass the ball as quickly as possible. However, compared with the shoulder pass, the passing height of the chest pass is lower and the player cannot pass the ball with the high speed between the long distance.

(a)

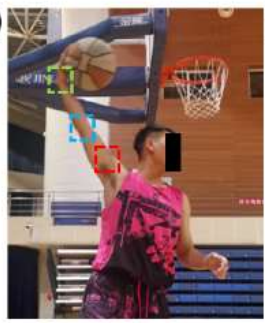

(e)

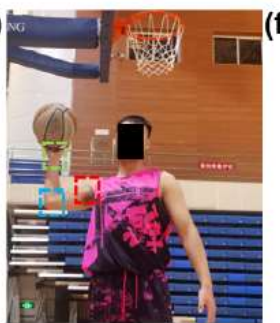

(i)

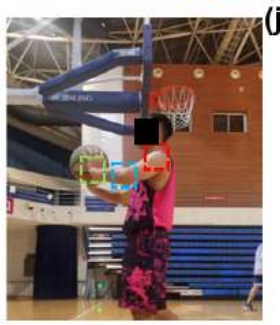

(b)
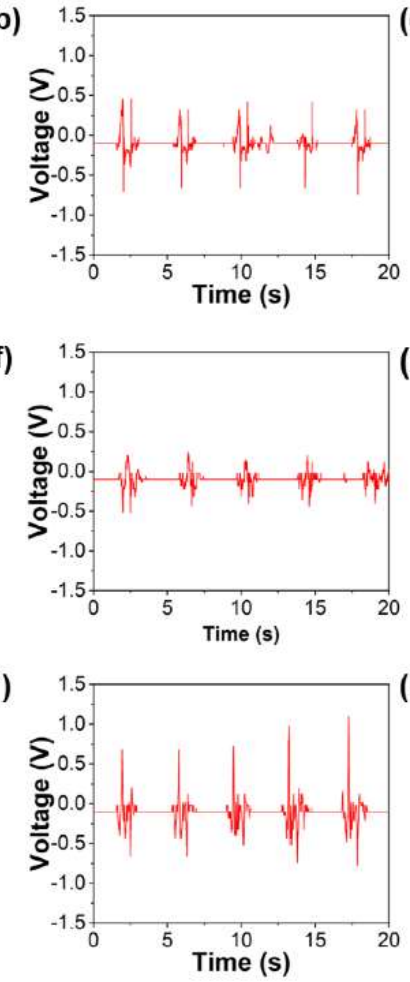

(c)

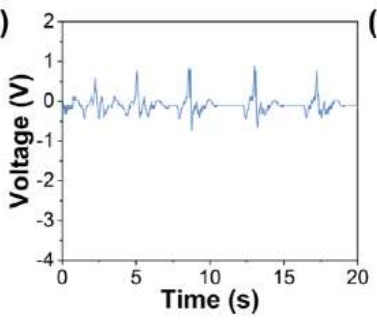

(g)

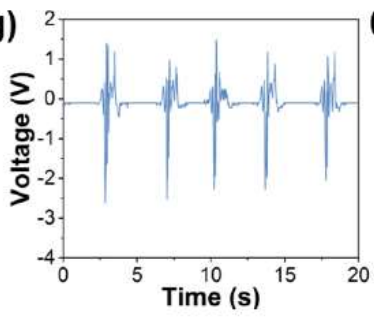

(k)

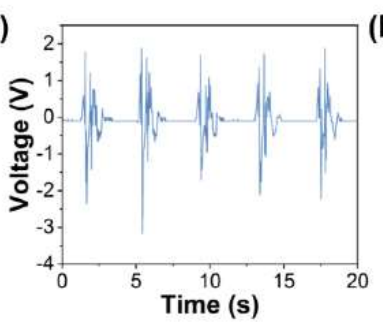

(d)

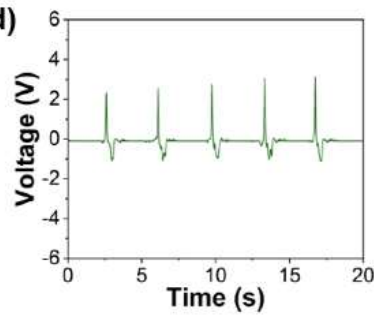

(h)
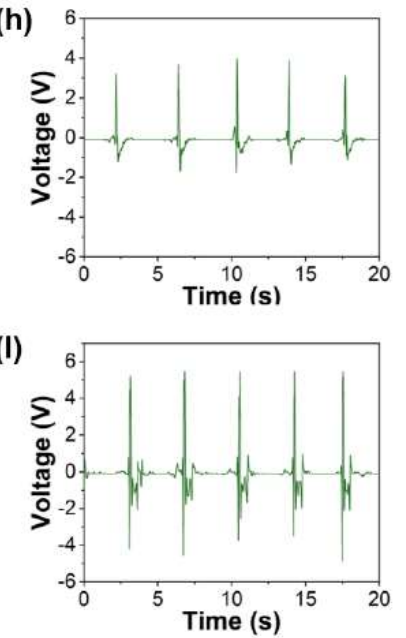

Figure 6. (a) Image of hook pass. (b-d) Outputting piezoelectric voltage of the shoulder, elbow, and wrist in hook pass motion. (e) Image of the shoulder pass. (f-h) Outputting piezoelectric voltage of the shoulder, elbow, and wrist in a shoulder pass motion. (i) Image of the chest pass. (j-1) Outputting piezoelectric voltage of the shoulder, elbow, and wrist in a chest pass motion.

We have made progress in motion monitoring. However, wireless transmission of information is still challenging. We need to combine computer with information science to realize PENG's wireless application in sports.

\section{Conclusions}

In summary, we report a transparent, stretchable, and biocompatible PENG which employed PAAM- $\mathrm{LiCl}$ hydrogel as the work electrode. The TSB-PEGN can sensitively detect changes of angle, twist, and frequency. Meanwhile, TSB-PEGN can keep a stable voltage output in a different environment. Moreover, TSB-PEGN can convert the mechanical energy of the human body into electrical energy, charge capacitor, Bluetooth etc. We demonstrate that the TSB-PEGN applies to the basketball technique. It can collect hook pass, shoulder pass, and chest pass motion information. It provides references for the 
athlete. The nanogenerator promotes PENG application and development, and shows its potential as a new generation of the motion monitoring system.

Supplementary Materials: The following are available online at https://www.mdpi.com/article/ 10.3390/electronics10212584/s1, Table S1: The advantages of PAAM-LiCl hydrogel compared with metal electrodes. Figure S1: Durability property of TSB-PENG. Movie S1: The output voltage signal of PENG at bend, twist and stretch, Movie S2: The PENG can charge $4.7 \mu \mathrm{F}$ capacitor and drive the calculator, Movie S3: The device can transmit wireless signal to control the LED, Movie S4: The output signal of the hook pass movement is collected, Movie S5: The output signal of the shoulder pass movement is collected, Movie S6: The output signal of the chest pass movement is collected.

Author Contributions: C.Z., Y.M. and C.J. put forward to the concept of the study. The data were collected, sorted out, and analyzed by Y.Z., T.Z., R.X. and C.J., Y.Z. and F.S. made the visualization. C.J. and Y.M. wrote the manuscript. C.Z. finished proof. funding acquisition, C.Z. All authors have read and agreed to the published version of the manuscript.

Funding: This research was funded by the Project supported by Fundamental Research Funds for the Central Universities: (N2021001).

Informed Consent Statement: Informed consent was obtained from all subjects involved in the study.

Data Availability Statement: The data presented in this study are available on request from the corresponding author.

Acknowledgments: The authors would like to thank the collaboration of all volunteers who participated in data collection.

Conflicts of Interest: The authors declare no conflict of interest.

\section{References}

1. Tian, X.; Lee, P.M.; Tan, Y.J.; Wu, T.L.; Yao, H.C.; Zhang, M.Y.; Li, Z.P.; Ng, K.A.; Tee, B.C.; Ho, J.S. Wireless body sensor networks based on metamaterial textiles. Nat. Electron. 2019, 2, 243-251. [CrossRef]

2. Kim, H.; Kim, Y.S.; Mahmood, M.; Kwon, S.; Zavanelli, N.; Kim, H.S.; Rim, Y.S.; Epps, F.; Yeo, W.H. Fully Integrated, Stretchable, Wireless Skin-Conformal Bioelectronics for Continuous Stress Monitoring in Daily Life. Adv. Sci. 2020, 7, 2000810. [CrossRef] [PubMed]

3. He, T.Y.; Wang, H.; Wang, J.H.; Tian, X.; Wen, F.; Shi, Q.F.; Ho, J.S.; Lee, C.K. Self-Sustainable Wearable Textile Nano-Energy Nano-System (NENS) for Next-Generation Healthcare Applications. Adv. Sci. 2019, 6, 1901437. [CrossRef] [PubMed]

4. Wei, J.J.; Wang, Z.Y.; Xing, X.P. A Wireless High-Sensitivity Fetal Heart Sound Monitoring System. Sensors 2021, 21, 193. [CrossRef] [PubMed]

5. Tang, Z.; Lin, S.Q.; Wang, Z.L. Quantifying Contact-Electrification Induced Charge Transfer on a Liquid Droplet after Contacting with a Liquid or Solid. Adv. Mater. 2021, 2102886. [CrossRef] [PubMed]

6. Zhang, J.; Lin, S.; Zheng, M.; Wang, Z.L. Triboelectric Nanogenerator as a Probe for Measuring the Charge Transfer between Liquid and Solid Surfaces. ACS Nano 2021, 15, 14830-14837. [CrossRef] [PubMed]

7. Xia, P.C.; Luo, J.J.; Wang, M.M. Adaptive compliant controller for space robot stabilization in postcapture phase. Proc. Inst. Mech. Eng. Part G J. Aerosp. Eng. 2021, 235, 937-948. [CrossRef]

8. Ning, C.; Dong, K.; Gao, W.C.; Sheng, F.F.; Cheng, R.W.; Jiang, Y.; Yi, J.; Ye, C.Y.; Peng, X.; Wang, Z.L. Dual-mode thermalregulating and self-powered pressure sensing hybrid smart fibers. Chem. Eng. J. 2021, 420, 129650. [CrossRef]

9. Luo, J.J.; Gao, W.C.; Wang, Z.L. The Triboelectric Nanogenerator as an Innovative Technology toward Intelligent Sports. Adv. Mater. 2021, 33, 2004178. [CrossRef] [PubMed]

10. Camomilla, V.; Bergamini, E.; Fantozzi, S.; Vannozzi, G. Trends Supporting the In-Field Use of Wearable Inertial Sensors for Sport Performance Evaluation: A Systematic Review. Sensors 2018, 18, 873. [CrossRef] [PubMed]

11. Mendes, J.J.A.; Vieira, M.E.M.; Pires, M.B.; Stevan, S.L. Sensor Fusion and Smart Sensor in Sports and Biomedical Applications. Sensors 2016, 16, 1569. [CrossRef]

12. Kos, A.; Umek, A. Smart sport equipment: SmartSki prototype for biofeedback applications in skiing. Pers. Ubiquit. Comput. 2018, 22, 535-544. [CrossRef]

13. Li, R.T.; Kling, S.R.; Salata, M.J.; Cupp, S.A.; Sheehan, J.; Voos, J.E. Wearable Performance Devices in Sports Medicine. Sports Health 2016, 8, 74-78. [CrossRef] [PubMed]

14. Luo, J.J.; Wang, Z.M.; Xu, L.; Wang, A.C.; Han, K.; Jiang, T.; Lai, Q.S.; Bai, Y.; Tang, W.; Fan, F.R.; et al. Flexible and durable wood-based triboelectric nanogenerators for self-powered sensing in athletic big data analytics. Nat. Commun. 2019, 10, 5147. [CrossRef] 
15. Mao, Y.P.; Zhu, Y.S.; Zhao, T.M.; Jia, C.J.; Bian, M.Y.; Li, X.X.; Liu, Y.G.; Liu, B.D. A Portable and Flexible Self-Powered Multifunctional Sensor for Real-Time Monitoring in Swimming. Biosensors 2021, 11, 147. [CrossRef]

16. Zhao, C.L.; Jia, C.J.; Zhu, Y.S.; Zhao, T.M. An Effective Self-Powered Piezoelectric Sensor for Monitoring Basketball Skills. Sensors 2021, 21, 5144. [CrossRef]

17. Mao, Y.P.; Zhu, Y.S.; Zhao, T.M.; Jia, C.J.; Wang, X.; Wang, Q. Portable Mobile Gait Monitor System Based on Triboelectric Nanogenerator for Monitoring Gait and Powering Electronics. Energies 2021, 14, 4996. [CrossRef]

18. Mao, Y.; Zhang, W.; Wang, Y.; Guan, R.; Liu, B.; Wang, X.; Sun, Z.; Xing, L.; Chen, S.; Xue, X. Self-Powered Wearable Athletics Monitoring Nanodevice Based on ZnO Nanowire Piezoelectric-Biosensing Unit Arrays. Sci. Adv. Mater. 2019, 11, 351-359. [CrossRef]

19. Mao, Y.P.; Shen, M.L.; Liu, B.; Xing, L.L.; Chen, S.; Xue, X.Y. Self-Powered Piezoelectric-Biosensing Textiles for the Physiological Monitoring and Time-Motion Analysis of Individual Sports. Sensors 2019, 19, 3310. [CrossRef]

20. Mao, Y.P.; Zhu, Y.S.; Jia, C.J.; Zhao, T.M.; Zhu, J.B. A Self-Powered Flexible Biosensor for Human Exercise Intensity Monitoring. J. Nanoelectron. Optoelectron. 2021, 16, 699-706. [CrossRef]

21. Xu, S.X.; Song, J. Study on the High-level Basketball Team Competence and Winning Methods. In Proceedings of the 2012 Third International Conference on Education and Sports Education (ESE 2012), Vol III 3rd International Conference on Education and Sports Education (ESE 2012), Macau, China, 1-2 April 2012; Singapore Management \& Sports Science INST PTE Ltd.: Singapore, 2012.

22. Zhou, X.J.; Gao, Z.; Zhang, Q.L. Analysis Approach of Winning Factors in Competitive Basketball. In Proceedings of the 2008 IEEE International Symposium on Knowledge Acquisition and Modeling Workshop Proceedings, Vols 1 and 2, International Symposium on Knowledge Acquisition and Modeling, Wuhan, China, 21-22 December 2008; IEEE: New York, NY, USA, 2008.

23. Papadimitriou, K.; Taxildaris, K.; Derri, V.; Mantis, K. Profile of different level basketball centers. J. Hum. Mov. Stud. 1999, 37, 87-105.

24. Sindik, J.; Nazor, D. Differences in Conative Characteristics and Perceived Group Cohesion of the Basketball Players Playing in Different Positions in the Team. Coll. Antropol. 2011, 35, 895-904. [PubMed]

25. Sekulic, D.; Pehar, M.; Krolo, A.; Spasic, M.; Uljevic, O.; Calleja-Gonzalez, J.; Sattler, T. Evaluation of Basketball-Specific Agility: Applicability of Preplanned and Nonplanned Agility Performances for Differentiating Playing Positions and Playing Levels. J. Strength Cond. Res. 2017, 31, 2278-2288. [CrossRef]

26. Stewart, J.C.; Yeh, S.C.; Jung, Y.B.; Yoon, H.J.; Whitford, M.; Chen, S.Y.; Li, L.; McLaughlin, M.; Rizzo, A.; Winstein, C.J. Intervention to enhance skilled arm and hand movements after stroke: A feasibility study using a new virtual reality system. J. Neuroeng. Rehabil. 2007, 4, 21. [CrossRef] [PubMed]

27. Struzik, A.; Pietraszewski, B.; Zawadzki, J. Biomechanical Analysis of the Jump Shot in Basketball. J. Hum. Kinet. 2014, 42, 73-79. [CrossRef] [PubMed]

28. Sosa, C.; Lorenzo, A.; Trapero, J.; Ribas, C.; Alonso, E.; Jimenez, S.L. Specific Absolute Velocity Thresholds during Male Basketball Games Using Local Positional System; Differences between Age Categories. Appl. Sci. 2021, 11, 4390. [CrossRef]

29. Malone, L.A.; Gervais, P.L.; Steadward, R.D. Shooting mechanics related to player classification and free throw success in wheelchair basketball. J. Rehabil. Res. Dev. 2002, 39, 701-709. [PubMed]

30. de Oliveira, G.C.M.; Carvalho, J.H.D.; Brazaca, L.C.; Vieira, N.C.S.; Janegitz, B.C. Flexible platinum electrodes as electrochemical sensor and immunosensor for Parkinson's disease biomarkers. Biosensors 2020, 152, 112016. [CrossRef]

31. Li, Y.T.; Miao, X.H.; Niu, L.; Jiang, G.M.; Ma, P.B. Human Motion Recognition of Knitted Flexible Sensor in Walking Cycle. Sensors 2020, 20, 35. [CrossRef]

32. Jiang, X.N.; Kim, K.; Zhang, S.J.; Johnson, J.; Salazar, G. High-Temperature Piezoelectric Sensing. Sensors 2014, 14, 144-169. [CrossRef]

33. Wooten, J.M.; Bevly, D.M.; Hung, J.Y. Piezoelectric Polymer-Based Collision Detection Sensor for Robotic Applications. Electronics 2015, 4, 204-220. [CrossRef]

34. Del-Rio-Ruiz, R.; Echevarria, J.J.; Eguiluz, X.; Lopez-Garde, J.M.; Legarda, J. Experimental Frequency Tuning Methodology of a Cantilever Piezoelectric Harvester Validated in a Multimodal Transportation. Electronics 2020, 9, 79. [CrossRef]

35. Jeong, S.Y.; Cho, J.Y.; Hong, S.D.; Hwang, W.; Jabbar, H.; Ahn, J.H.; Jhun, J.P.; Sung, T.H. Self-Powered Operational Amplifying System with a Bipolar Voltage Generator Using a Piezoelectric Energy Harvester. Electronics 2020, 9, 41. [CrossRef]

36. Wang, D.W.; Fan, Z.M.; Rao, G.H.; Wang, G.; Liu, Y.; Yuan, C.L.; Ma, T.; Li, D.J.; Tan, X.L.; Lu, Z.L.; et al. Ultrahigh piezoelectricity in lead-free piezoceramics by synergistic design. Nano Energy 2020, 76, 104944. [CrossRef]

37. He, Y.X.; Yang, Q.; Luo, M.D.; Liu, R.Y. Effect of the physical parameters of longitudinally polarized PZT tubes on PZT sensors. J. Phys. D Appl. Phys. 2020, 53, 275501. [CrossRef]

38. Li, F.; Cabral, M.J.; Xu, B.; Cheng, Z.X.; Dickey, E.C.; LeBeau, J.M.; Wang, J.L.; Luo, J.; Taylor, S.; Hackenberger, W.; et al. Giant piezoelectricity of $\mathrm{Sm}$-doped $\mathrm{Pb}(\mathrm{Mg} 1 / 3 \mathrm{Nb} 2 / 3) \mathrm{O}-3-\mathrm{PbTiO} 3$ single crystals. Science 2019, 364, 264-268. [CrossRef]

39. Qiu, C.R.; Wang, B.; Zhang, N.; Zhang, S.J.; Liu, J.F.; Walker, D.; Wang, Y.; Tian, H.; Shrout, T.R.; Xu, Z.; et al. Transparent ferroelectric crystals with ultrahigh piezoelectricity. Nature 2020, 577, 350-354. [CrossRef]

40. Park, K.I.; Jeong, C.K.; Ryu, J.; Hwang, G.T.; Lee, K.J. Flexible and Large-Area Nanocomposite Generators Based on Lead Zirconate Titanate Particles and Carbon Nanotubes. Adv. Energy Mater. 2013, 3, 1539-1544. [CrossRef] 
41. Sato, K.; Kang, W.H.; Saga, K.; Sato, K.T. Biology of sweat glands and its disorders. I. Normal sweat gland function. J. Am. Acad. Dermatol. 1989, 20, 713-726. [CrossRef]

42. Raiszadeh, M.M.; Ross, M.M.; Russo, P.S.; Schaepper, M.A.; Zhou, W.D.; Deng, J.H.; Ng, D.; Dickson, A.; Dickson, C.; Strom, M.; et al. Proteomic Analysis of Eccrine Sweat: Implications for the Discovery of Schizophrenia Biomarker Proteins. J. Proteome Res. 2012, 11, 2127-2139. [CrossRef]

43. Manjakkal, L.; Pullanchiyodan, A.; Yogeswaran, N.; Hosseini, E.S.; Dahiya, R. A Wearable Supercapacitor Based on Conductive PEDOT:PSS-Coated Cloth and a Sweat Electrolyte. Adv. Mater. 2020, 32, 1907254. [CrossRef] [PubMed]

44. Wang, J.Y.; Liu, W.H.; Moffit, J. Skills and Offensive Tactics Used in Pick-up Basketball Games. Percept. Motor Skill 2009, 109, 473-477. [CrossRef] [PubMed] 